\title{
UNCOMMON DITERPENES WITH THE SKELETON OF SIX-FIVE-SIX FUSED-RINGS FROM TAIWANIA CRYPTOMERIOIDES
}

\author{
WANG-Hong Lin, Jim-Min FANG and YU-Shia Cheng* \\ Department of Chemistry, National Taiwan University, Taipei, Taiwan 106, Republic of China
}

(Received in revised form 21 March 1995)

Key Word Index-Taiwania cryptomerioides, taxodiaceae; leaves; diterpenes.

Abstract-Four diterpenoid aldehydes and one norditerpenoid ketone having the uncommon skeleton of six-five-six fused-rings were isolated from the leaves of Taiwania cryptomerioides.

\section{INTRODUCTION}

Taiwania cryptomerioides Hayata, is an endemic evergreen species with thick linear-triangular leaves and elongate ovoid cones. The chemical constituents of this plant have been investigated extensively [1-5]. Varied sesquiterpenes, lignans and bisflavones have been found in the essential oil of the leaves and wood. We report herein the isolation of four diterpenes $1-4$ and a norditerpene 5 , which have the uncommon skeleton of fused 6-5-6 rings, in addition to known diterpenes.

\section{RESULTS AND DISCUSSION}

Four known abietane-type diterpenes, ferruginol (6) $[6,7], 6,7$-dehydroferruginol (7) $[6], 7 \beta$-hydroxyroyleanone (8) [8], 7 $\alpha$-hydroxyroyleanone (9) [8] and a secoabietane dialdehyde (10) [9], were identified by comparison of their physical and spectral data (mp, $[\alpha]$, mass, IR, ${ }^{1} \mathrm{H}$ and ${ }^{13} \mathrm{C}$ NMR) with those in the literature.

Compound 1 , namely taiwaniaquinone $A$, was isolated as orange crystals, $\mathrm{mp} 201-203^{\circ}$ (decomposed). Its structure was determined by spectral methods. The exact mass $[\mathrm{M}]^{+}$at $\mathrm{m} / \mathrm{z} 330.1825$ indicated the molecular formula $\mathrm{C}_{20} \mathrm{H}_{26} \mathrm{O}_{4}$. The IR absorptions at 1659 and $1639 \mathrm{~cm}^{-1}$, and the UV absorptions at 432 ( $\varepsilon 579), 284(\varepsilon 6400)$ and $207 \mathrm{~nm}(\varepsilon 12600)$ were attributable to the quinone moiety. The ${ }^{13} \mathrm{C}$ signals of the quinone moiety occurred at $\delta 124.6,149.2,151.2,152.8,181.0$ and 185.3 (Table 1). The proton resonance (Table 2) at $\delta 9.84(d, J=4 \mathrm{~Hz})$ and the carbon signal at $\delta 200.2$ were attributable to an aldehyde group. The skeleton of 6-5-6 fused-rings were established by means of the $\mathrm{C}-\mathrm{H}$ COSY and the HMBC experiment. Irradiation of Me-10 (at $\delta 1.14$ ) caused $10 \% \mathrm{nOe}$ of $\mathrm{H}-7$ (at $\delta 3.76$ ). The stereochemistry of 1 was thus confirmed.

\footnotetext{
*Author to whom correspondence should be addressed.
}

H-5 and H-7 were trans oriented and had a large coupling constant $11.5 \mathrm{~Hz}$. Compound $1,[\alpha]_{\mathrm{D}}^{25}-220.6^{\circ}$, showed a positive Cotton effect with the maximum at $309 \mathrm{~nm}([\theta], 22600)$. Compound 1 was assigned to have the $(5 S, 7 R, 10 S)$-configuration by analogy to those abietanes found in the plants of the Taxodiaceae family $[4,5]$. The uncommon 6-5-6 rings skeleton was presumably formed (biogenetically) from the pinacol rearrangement of abietane-6,7-diol as depicted in A (Scheme 1).

Table $1 .{ }^{13} \mathrm{CNMR}$ spectral data of 1-5 ( $\delta$ value in ppm)

\begin{tabular}{|c|c|c|c|c|c|}
\hline C & $1^{*}$ & $2+$ & $3+$ & $4^{*}+$ & $5^{*} \S$ \\
\hline 1 & 34.4 & 35.8 & 37.6 & 35.6 & 30.3 \\
\hline 2 & 19.3 & 20.2 & 20.4 & 19.7 & 17.5 \\
\hline 3 & 41.1 & 44.1 & 44.2 & 41.7 & 36.5 \\
\hline 4 & 33.6 & 35.1 & 35.2 & 34.0 & 34.3 \\
\hline 5 & 61.5 & 62.1 & 69.6 & 61.5 & 65.1 \\
\hline 6 & 200.2 & 203.7 & 201.5 & 205.0 & - \\
\hline 7 & 54.4 & 88.4 & 90.2 & 54.5 & 211.1 \\
\hline 8 & 149.2 & 148.8 & 147.4 & 113.4 & 118.3 \\
\hline 9 & 152.8 & 155.1 & 154.3 & 132.3 & 142.7 \\
\hline 10 & 48.7 & 50.2 & 47.3 & 46.3 & 42.7 \\
\hline 11 & 181.0 & 182.8 & 182.6 & 135.2 & 138.4 \\
\hline 12 & 151.2 & 154.3 & 153.5 & 146.8 & 152.2 \\
\hline 13 & 124.6 & 125.0 & 125.6 & 116.7 & 126.1 \\
\hline 14 & 185.3 & 186.1 & 187.0 & 146.0 & 151.1 \\
\hline 15 & 24.2 & 24.9 & 24.9 & 25.1 & 25.9 \\
\hline 16 & 19.8 & 20.4 & 20.5 & 21.0 & 20.6 \\
\hline 17 & 19.8 & 20.4 & 20.5 & 21.1 & 20.6 \\
\hline 18 & 35.0 & 34.8 & 33.8 & 34.4 & 24.4 \\
\hline 19 & 21.8 & 23.7 & 24.6 & 22.0 & 33.0 \\
\hline 20 & 20.1 & 22.8 & 23.9 & 22.4 & 28.8 \\
\hline
\end{tabular}

* Solution in $\mathrm{CDCl}_{3}$.

† Solution in acetone- $d_{6}$.

¥ The signal of methylenedioxy group in 4 appeared at $\delta 100.8$. $\S$ The signal of methoxyl group in 5 appeared at $\delta 62.1$. 
Table 2. ${ }^{1} \mathrm{H}$ NMR spectral data of 1-5 ( $\delta$ value in ppm; $J$ value in $\mathrm{Hz}$ )

\begin{tabular}{rlllll}
\hline H & \multicolumn{1}{c}{$\mathbf{1}^{*}$} & \multicolumn{1}{c}{$2 \dagger$} & \multicolumn{1}{c}{$3 \dagger$} & \multicolumn{1}{c}{$\mathbf{4}^{*} \ddagger$} & \multicolumn{1}{c}{$\mathbf{5}^{*} \S$} \\
\hline 5 & $2.13(d, J=11.5)$ & $2.04(s)$ & $2.26(s)$ & $2.16(d, J=11)$ & $2.10(s)$ \\
6 & $9.84(d, J=4)$ & $9.82(s)$ & $10.08(s)$ & $9.47(d, J=5)$ & \\
7 & $3.76(d d, J=11.5,4)$ & & & $3.77(d d, J=11,5)$ & \\
15 & $3.12($ sept,$J=7)$ & $3.12($ sept,$J=7)$ & $3.14(s e p t, J=7)$ & $3.16(s e p t, J=7)$ & $3.25($ sept, $J=7)$ \\
16 & $1.17(d, J=7)$ & $1.16(d, J=7)$ & $1.18(d, J=7)$ & $1.26(d, J=7)$ & $1.36(d, J=7)$ \\
17 & $1.18(d, J=7)$ & $1.16(d, J=7)$ & $1.17(d, J=7)$ & $1.26(d, J=7)$ & $1.36(d, J=7)$ \\
18 & $0.79(s)$ & $0.78(s)$ & $1.11(s)$ & $0.89(s)$ & $0.86(s)$ \\
19 & $1.05(s)$ & $1.20(s)$ & $1.04(s)$ & $1.06(s)$ & $1.24(s)$ \\
20 & $1.14(s)$ & $1.49(s)$ & $1.35(s)$ & $1.10(s)$ & $1.42(s)$ \\
\hline
\end{tabular}

* Solution in $\mathrm{CDCl}_{3}$.

† Solution in acetone- $d_{6}$.

¥The signals of methylenedioxy group in 4 appeared at $5.82(d, J=1)$ and $5.87(d, J=1)$.

$\S$ The signal of methoxyl group in 5 appeared at $\delta 3.78(s)$.
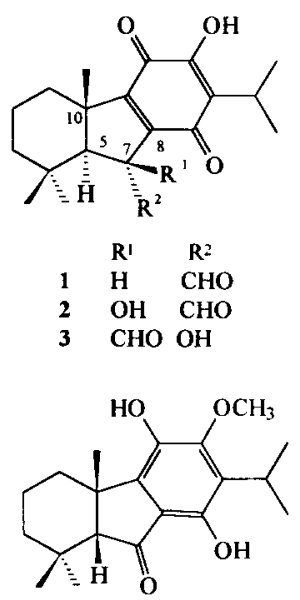

5<smiles>CC(C)c1cc2c(cc1O)[C@]1(C)CCCC(C)[C@@H]1CC2</smiles>

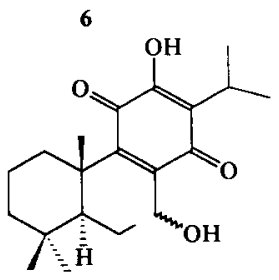

$87 \beta-\mathrm{OH}$

$97 \alpha-\mathrm{OH}$
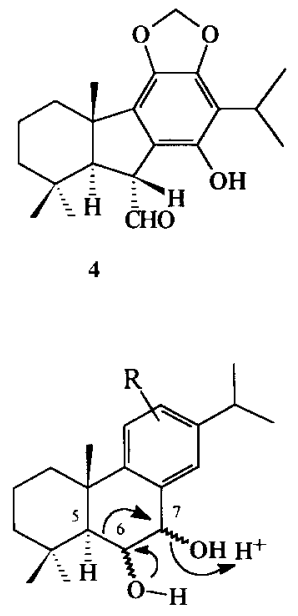

$\mathbf{A}$
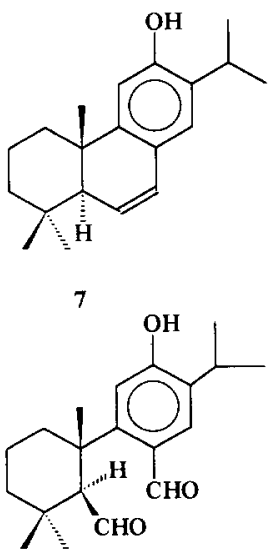

10

The structures of two 7-hydroxylated derivatives 2 (namely taiwaniaquinone $B$ ) and 3 (namely taiwaniaquinone $\mathrm{C}$ ) were determined by similar procedures. Their pertinent ${ }^{13} \mathrm{C}$ and ${ }^{1} \mathrm{H}$ signals are listed in Tables 1 and 2. The formyl group in 3 oriented on the $\beta$-face as the proton signal at $\delta 10.08$ showed $10 \% \mathrm{nOe}$ upon irradiation of $\mathrm{Me}-10$ (at $\delta 1.35$ ). Compound 2 showed a positive Cotton effect with maximum $[\theta]_{304}$ 21 100, whereas the epimer 3 showed a negative Cotton effect with minimum $[\theta]_{316}-6100$.

The molecular formula $\mathrm{C}_{21} \mathrm{H}_{28} \mathrm{O}_{4}$ for 4 , which we have named taiwaniaquinol $\mathrm{A}$, was deduced from its parent peak at $m / z$ 344.1979. Compound 4 was colourless and crystalline, $\mathrm{mp} 158-160^{\circ} \mathrm{C}$. It showed intense IR absorptions at 3335 and $1686 \mathrm{~cm}^{-1}$ for the hydroxyl and carbonyl groups. The ${ }^{1} \mathrm{H}$ NMR spectrum exhibited characteristic signals at $\delta 5.87$ and 5.82 for the methylenedioxy group. The corresponding carbon signal appeared at $\delta 100.8$. The structure of taiwaniaquinol $\mathbf{A}(4)$ was finally determined by means of the $\mathrm{H}-\mathrm{H}$ COSY, $\mathrm{C}-\mathrm{H}$ COSY and HMBC experiments. The nOe effect $(13 \%)$ of $\mathrm{H}-7$, observed by irradiation of Me-10 (at $\delta 1.10$ ), was in agreement with the assigned stereochemistry. The large coupling constant $11 \mathrm{~Hz}$ between $\mathrm{H}-5$ and H-7 conformed to their trans-relationship.

The NMR analysis revealed that compound $5\left(\mathrm{C}_{20} \mathrm{H}_{28} \mathrm{O}_{4}\right)$ is a norditerpene containing a methoxyl group $\left(\delta_{\mathrm{H}} 3.78\right.$ and $\left.\delta_{\mathrm{C}} 62.1\right)$. The conjugated ketone showed IR absorption at $1638 \mathrm{~cm}^{-1}$ and a carbon signal at $\delta$ 211.1. Compound 5 , named taiwaniaquinol $\mathbf{B}$, has a cis-ring junction as revealed by a nOe experiment, i.e. an $18 \%$ enhancement of $\mathrm{H}-5$ (at $\delta 2.10$ ) resulting from irradiation of Me-10 (at $\delta 1.42$ ).

In summary, compounds $1-4$ represent a new class of diterpenes, and $\mathbf{5}$ is a novel norditerpene. This is the first report of compounds having the uncommon 6-5-6 rings in nature.

Scheme 1. 


\section{EXPERIMENTAL}

General and plant material. The dried leaves $(1.75 \mathrm{~kg})$ of $T$. cryptomerioides were exhaustively extracted with $\mathrm{Me}_{2} \mathrm{CO}(71 \times 3)$. The combined extracts were concd to $\mathrm{ca}$ 0.81 , and taken up with $\mathrm{CHCl}_{3}(0.81 \times 3)$. The $\mathrm{CHCl}_{3}$ soluble portion was concd $(55 \mathrm{~g})$ and subjected to silica gel CC. The portion obtained from elution with EtOAc in hexane ( $5-20 \%$ ) was further purified by HPLC (Hibar Lichrosorb Si 60,7 or $10 \mu \mathrm{m}, 25 \times 1 \mathrm{~cm}$ ) with elution by EtOAc-hexane $(1: 10)$ to give compounds 1 (40 mg), $2(20 \mathrm{mg}), 3(20 \mathrm{mg}), 4(25 \mathrm{mg}), 5(10 \mathrm{mg}), 6(30 \mathrm{mg})$, $7(6 \mathrm{mg}), 8$ (251 mg), $9(55 \mathrm{mg}), 10$ (15 mg). Merck Silica gel $60 \mathrm{~F}$ sheets were used for analyt. TLC (EtOAc-hexane, 1:9).

Taiwaniaquinone $A$ (1). Orange crystals from EtOAchexane (1:9). Mp 201-203 ${ }^{\circ} \mathrm{C}$ (decomposed). [ $\left.\alpha\right]_{\mathrm{D}}^{25}-$ $220.6^{\circ}\left(\mathrm{CHCl}_{3} ; c\right.$ 1.3). TLC (EtOAc-hexane, $\left.1: 9\right) \boldsymbol{R}_{\boldsymbol{f}} 0.5$. IR $v_{\max }^{\mathrm{KBr}} \mathrm{cm}^{-1} .3331,1706,1659,1639$. UV $\lambda_{\max }^{\mathrm{MeOH}} \mathrm{nm}(\varepsilon)$ : 432 (579), 284 (6400), 207 (12 600). EIMS (70 eV) $\mathrm{m} / \mathrm{z}$ (rel. int.): $330[\mathrm{M}]^{+}(2), 302$ (100), 287 (22), 233 (68), 220 (35), 121 (5), 109 (8). HRMS for $\mathrm{C}_{20} \mathrm{H}_{26} \mathrm{O}_{4}$ requires: 330.1832. Found: 330.1825 . CD (MeOH) $[\theta]_{309}+22600,[\theta]_{277}-$ $1900,[\theta]_{267}-1100,[\theta]_{249}-4400,[\theta]_{237}-3600$

Taiwaniaquinone $B(2)$. Orange crystals from EtOAchexane (1:9). Mp $183-185^{\circ} \mathrm{C}$ (decomposed). $[\alpha]_{\mathrm{D}}^{31}-83^{\circ}$ $\left(\mathrm{CHCl}_{3} ; c\right.$ 0.27). TLC (EtOAc-hexane, $\left.1: 9\right) \boldsymbol{R}_{\boldsymbol{f}}$ 0.43. IR $v_{\max }^{\mathrm{KBr}} \mathrm{cm}^{-1}: 3498,3376,1720,1662,1664$. UV $\lambda_{\max }^{\mathrm{MeOH}} \mathrm{nm}$ (ع): 427 (619), 277 (6000), 204 (8900). EIMS (70 eV) m/z (rel. int.): 317 [M - CHO $]^{+}$(56), 299 (4), 209 (100), 187 (5), 115 (10), 109 (68), HRMS for $\mathrm{C}_{20} \mathrm{H}_{26} \mathrm{O}_{5}-\mathrm{CHO}$ requires: 317.1754. Found: 317.1749. $\mathrm{CD}(\mathrm{MeOH})$ $[\theta]_{353}+1800,[\theta]_{304}+21100,[\theta]_{257}-9800$.

Taiwaniaquinone $C$ (3). Orange crystals from EtOAchexane (1:9). Mp 210-212 ${ }^{\circ} \mathrm{C}$ (decomposed). $[\alpha]_{\mathrm{D}}^{31}-$ $266\left(\mathrm{CHCl}_{3} ; c 0.27\right)$. TLC (EtOAc-hexane, $\left.1: 9\right) R_{f} 0.45$. IR $v_{\max }^{\mathrm{KBr}} \mathrm{cm}^{-1}: 3497,3358,1718,1648,1623$. UV $\lambda_{\max }^{\mathrm{MeOH}} \mathrm{nm}$

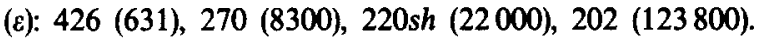
EIMS (70 eV) $\mathrm{m} / z$ (rel. int.): 317 [M $-\mathrm{CHO}^{+}$(52), 299 (4), 233 (5), 209 (100), 205 (8), 115 (9), 109 (68). HRMS for $\mathrm{C}_{20} \mathrm{H}_{26} \mathrm{O}_{5}-\mathrm{CHO}$ requires: 317.1754 . Found: 317.1749 . $\mathrm{CD}(\mathrm{MeOH})[\theta]_{399}+2600,[\theta]_{352}+6200,[\theta]_{316}-$ $6100,[\theta]_{280}+17700,[\theta]_{248}+4000,[\theta]_{228}+12000$.

Taiwaniaquinol $A$ (4). Crystals from EtOAc-hexane (1:9). Mp $158-160^{\circ} \mathrm{C} .[\alpha]_{\mathrm{D}}^{31}+88.2^{\circ}\left(\mathrm{CHCl}_{3} ; c 0.64\right)$. TLC (EtOAc-hexane, 1:9) $\boldsymbol{R}_{\boldsymbol{f}} \mathbf{0 . 4 8}$. IR $\boldsymbol{v}_{\max }^{\mathrm{KBr}} \mathrm{cm}^{-1}$. 3335, 1686. UV $\lambda_{\max }^{\mathrm{MeOH}} \mathrm{nm}(\varepsilon): 293$ (4300), 206 (34 200). EIMS (70 eV) $m / z$ (rel. int.): 344 (82), 315 (100), 273 (3), 259 (10), 245 (25), 231 (10), 203 (4). HRMS for $\mathrm{C}_{21} \mathrm{H}_{28} \mathrm{O}_{4}$ requires: 344.1988. Found: 344.1979 . CD (MeOH) $[\theta]_{352}+200$, $[\theta]_{308}+16800,[\theta]_{280}-6400,[\theta]_{260}-2500$, $[\theta]_{251}-2800,[\theta]_{226}+2000,[\theta]_{210}-1000$.
Taiwaniaquinol $B$ (5). Crystals from EtOAc-hexane (1:9). Mp $142-144^{\circ} \mathrm{C} .[\alpha]_{\mathbb{D}}^{31}-37.7^{\circ}\left(\mathrm{CHCl}_{3} ; c 0.27\right)$. TLC (EtOAc-hexane, 1:9) $R_{f} 0.63$. IR $v_{\max }^{\mathrm{KBr}} \mathrm{cm}^{-1}: 3297$, 1638. UV $\lambda_{\max }^{\mathrm{MeOH}} \mathrm{nm}(\varepsilon): 350$ (4900), 276 (10000), 238 (14000), 207 (18 700). EIMS (70 eV) $\mathrm{m} / z$ (rel. int.): 332 (100), 317 (38), 263 (8), 249 (45), 233 (10), 219 (6), 149 (3). HRMS for $\mathrm{C}_{20} \mathrm{H}_{28} \mathrm{O}_{4}$ requires: 332.1988. Found: 332.1986 .

Ferruginol (6). Oil solid. $[\alpha]_{\mathrm{D}}^{20}+37^{\circ}\left(\mathrm{CHCl}_{3} ; \mathrm{c} \mathrm{1.2)}\right.$ (lit. $\left.[6][\alpha]_{\mathrm{D}}^{16}+40.6^{\circ}\right)$.

6,7-Dehydroferruginol (7). Oil. $[\alpha]_{\mathrm{D}}^{24}-59^{\circ}\left(\mathrm{CHCl}_{3}\right.$; c 0.5$)$ (lit. $[6][\alpha]_{\mathrm{D}}-60^{\circ}$ ).

$7 \beta$-Hydroxyroyleanone (8). Yellow solid. Mp $210-212^{\circ} \mathrm{C}\left(\right.$ lit. $\left.[10,11] 212-214^{\circ}\right) .[\alpha]_{\mathrm{D}}^{20}+330^{\circ}\left(\mathrm{CHCl}_{3}\right.$; c 1.3) $\left\{\right.$ lit. $\left.[10,11][\alpha]_{\mathrm{D}}+340^{\circ}\left(\mathrm{CHCl}_{3}\right)\right\}$.

$7 \alpha$-Hydroxyroyleanone (9). Yellow solid. Mp 175$176^{\circ} \mathrm{C}$ (lit. [12] 173-175 $) .[\alpha]_{\mathrm{D}}^{22}-135^{\circ}\left(\mathrm{CHCl}_{3} ; c\right.$ 1.6) [lit. $\left.[12][\alpha]_{\mathrm{D}}+132^{\circ}\left(\mathrm{CHCl}_{3}\right)\right]$.

12-Hydroxy-6,7-secoabieta-8,11,13-triene-6,7-dial (10). Solid. Mp 189-191 ${ }^{\circ} \mathrm{C}$ (lit. [9] 191-192 ${ }^{\circ}$ ). [ $\left.\alpha\right]_{\mathrm{D}}^{30}+20^{\circ}$ (MeOH; $c$ 0.7) $\left\{(\text { lit. [9] [ } \alpha]_{\mathrm{D}}^{25}+22^{\circ}(\mathrm{MeOH} ; c\right.$ 1.2) $\}$.

Acknowledgement-We thank the National Science Council for financial support.

\section{REFERENCES}

1. Fang, J.-M. and Cheng, Y.-S. (1992) J. Chin. Chem. Soc. (Taipei) 39, 647.

2. Kamil, M., Ilyas, M., Rahman, W., Hasaka, N., Okigawa, M. and Kawano, N. (1981) J. Chem. Soc., Perkin Trans. 1. 553.

3. Kuo, Y.-H., Chen, W.-C. and Lin, Y.-T. (1987) Chem. Express 2, 105.

4. Su, W.-C., Fang, J.-M. and Cheng, Y.-S. (1993) Phytochemistry 34, 779.

5. Su, W.-C., Fang, J.-M. and Cheng, Y.-S. (1994) Phytochemistry 35, 1279.

6. Bredenberg, J. B. (1957) Acta Chem. Scand. 11, 932.

7. Lin, Y.-T., Kuo, Y.-H. and Chang, B.-H. (1975) J. Chin. Chem. Soc. $22,331$.

8. Hensch, M., Ruedi, P. and Eugster, C. H. (1975) Helv. Chim. Acta 58, 1921.

9. Fang, J.-M., Jan, S.-T. and Cheng, Y.-S. (1986) J. Chem. Res. (S) 350.

10. Kupchan, S. M., Karim, A. and Marcks, C. (1969) J. Org. Chem. 34, 3912.

11. Kupchan, S. M., Karim, A. and Marcks, C. (1968) J. Am. Chem. Soc. 90, 5923.

12. Edwards, O. E., Feniak, G. and Los, M. (1962) Can. J. Chem. 40, 1542. 